\title{
Métrica de Hausdorff en el Ambiente Difuso
}

\section{Hausdorff Metric in the Fuzzy Environment}

\section{Carlos Orlando Ochoa Castillo ${ }^{1}$ Laura Victoria Forero Vega ${ }^{2}$}

${ }^{1}$ Universidad Distrital Francisco José de Caldas, correo electrónico: oochoac@udistrital.edu.co,

${ }^{2}$ Universidad Distrital Francisco José de Caldas, correo electrónico: lau_forero85@ hotmail.com

Recibido: 28-04-2015. Modificado: 01-09-2015. Aceptado: 26-07-2016

\section{Resumen}

Contexto: De manera intuitiva, se ha establecido el concepto de conjunto como una colección distinta de elementos, esto es, un conjunto se determina vía la relación de pertenencia de un elemento de un universo al conjunto. La situación, por supuesto, es si pertenece o no pertenece; en un subconjunto difuso a cada elemento del universo se le asocia con un grado de pertenencia, que es un número entre 0 y 1. Los subconjuntos difusos se establecen como una correspondencia entre cada elemento del universo y un grado de pertenencia.

Método: El estudio fue basado en trabajos anteriores como artículos o libros, en donde autores exponen ideas sobre la importancia de los subconjuntos difusos y la necesidad de crear con ellos nuevas teorías y espacios.

Resultados: Al combinar dos teorías, se genera un nuevo ambiente de estudio que permite afirmar que la distancia de Hausdorff corresponde, extiende y ajusta la nocion de distancia entre subconjuntos no vacíos compactos en el ambiente de los espacios métricos, mas exactamente en $\left(\mathbb{R}^{n}, d_{u}\right)$.

Conclusiones: La construcción realizada permite obtener un espacio métrico con varias cualidades, en donde se puede afirmar que son consequencia del objeto de estudio inicial.

Palabras clave: Conjuntos compactos, conjuntos difusos, métrica de Hausdorff.

Idioma: Español

\section{Abstract}

Context: Intuitively, the concept the set has been established as a collection of different elements, that is, a set is determined via the relationship of membership of an element of a universe as a whole. The situation, of course, is whether or does not belong; in a diffuse to each element subset of the universe it is associated with a degree of membership, which is a number between 0 and 1 . The fuzzy subsets are established as a correspondence between each element of the universe and a degree of membership.

Method: The study was based on previous work as articles or books, where authors present ideas about the importance of fuzzy subsets and the need to create with them new theories and spaces.

Results: By combining two theories, a new study environment that allows state that corresponds Hausdorff distance, extends and adjusts the notion of distance between nonempty compact subsets in the environment of metrics spaces, more accurately generated in $\left(\mathbb{R}^{n}, d_{u}\right)$.

Conclusions: The construction carried out allows a metric space with several qualities, where we can say that are the object consequence initial study.

Keywords: Compact sets, fuzzy sets, Hausdorff metric.

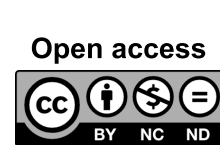

Citación: C.O. Ochoa, L.V. Forero, "Métrica de Hausdorff en el Ambiente Difuso," INGENIERÍA, vol. 21, no. 3, pp. 346-359, 2016.

(c) Los autores; titular de derechos de reproducción Universidad Distrital Francisco José de Caldas. En línea DOI: http://dx.doi.org/10.14483/udistrital.jour.reving.2016.3.a06 


\section{Introducción}

En 1965 Zadeh introdujo la noción de conjunto difuso (ver [12]) debido a que la mayoria de la veces, las clases de cosas encontradas en el mundo fisico real no tienen precisamente un criterio de pertenencia. Esta observación pone en conexión la existencia de las representaciones mentales de la realidad y representaciones matemáticas habituales de los mismos y fue el punto de partida hacia el desarrollo de los conjuntos difusos.

En 1980 Dubois y Prade definieron las distancia entre dos conjuntos difusos (ver [4]); luego Puri y Ralescu en 1983 expusieron una introducción de utilizar la distancia de Hausdorff entre conjuntos difusos; aunque la mayor referencia fue expuesta por Diamond y Kloeden [3] en 1994. Lo anterior condujo a que más adelante y mientras se investigaba problemas de sistemas dinamicos sin solución, Laksmikanthan y R.N Mohapatra [8] en 2003 publicaron lo hecho por Diamond y Kloeden para $\mathbb{R}^{n}$ como medio de resolver dichos problemas.

Este artículo tiene como finalidad el estudio de la métrica de Hausdorff, construída inicialmente en el ambiente $\mathbb{R}^{n}$ y luego extendida a una clase particular de subconjuntos difusos de $\mathbb{R}^{n}$, obteniendo un nuevo espacio métrico. Se parte de una definición de distancia entre un punto y un conjunto, con ello se edifica paso a paso la métrica de Hausdorff, igualmente se exhibe para el ambiente difuso.

En la primera sección, Métrica de Hausdorff, se muestra la construcción del espacio métrico $\left(K_{n}, d_{H}\right)$, de los subconjuntos compactos de $\mathbb{R}^{n}$ con la métrica de Hausdorff y se exponen algunas de sus propiedades; en la sección El espacio $E^{n}$, se describe un espacio particular de conjuntos difusos de $\mathbb{R}^{n}$ y algunas características; en la tercera sección, El espacio métrico $\left(E^{n}, d\right)$, se muestra el espacio resultante al relacionar la teoría de las dos secciones anteriores; en la última sección, Comparación con otros espacios métricos, se exponen otras distancias definidas en subconjuntos difusos y se realiza una comparación con el trabajo realizado anteriormente.

\section{Métrica de Hausdorff}

Apartir del espacio métrico $\left(\mathbb{R}^{n}, d_{u}\right)$, es decir, para $x=\left(x_{1}, x_{2}, \ldots, x_{n}\right)$ y $z=\left(z_{1}, z_{2}, \ldots, z_{n}\right)$ elementos de $\mathbb{R}^{n}, d_{u}(x, z)=\|x-z\|=\sqrt{\sum_{k=1}^{n}\left(x_{k}-z_{k}\right)^{2}}$, [7] , [1]; se inicia la construcción de la métrica de Hausdorff.

Definición 2.1. Sea $x$ un punto de $\mathbb{R}^{n}$ y $A$ un subconjunto no vacío de $\mathbb{R}^{n}$, la distancia $d(x, A)$ del punto $x$ a $A$ es

$$
d(x, A)=\inf \left\{d_{u}(x, a): a \in A\right\} .
$$

En los espacios métricos, se tienen diferentes tipos de colecciones de sus elementos de acuerdo a unas condiciones, estos son, entre otros, los conceptos de vecindad, bola abierta, bola cerrada y adherencia, que son como en [1].

Proposición 2.1. Sea $x$ un punto en $\mathbb{R}^{n}$ y $A$ un subconjunto no vacío de $\mathbb{R}^{n}$ entonces:

1. $d(x, A) \geq 0$, 
2. $d(x, A)=0$ si y solo si $x \in \bar{A}$

Prueba.

1. Por $\operatorname{ser}\left(\mathbb{R}^{n}, d_{u}\right)$ un espacio métrico, $d_{u}(x, a) \geq 0$ para todo $a \in A$, luego por propiedades del ínfimo, ínf $\{\|x-a\|: a \in A\} \geq 0$, así $d(x, A) \geq 0$.

2. Sea $d(x, A)=0$ y se supone que $x \notin \bar{A}$, esto es, existe $\epsilon>0$ tal que $\mathbb{B}_{\epsilon}(x) \cap A=\emptyset$ lo que indica que $d(x, A)>\epsilon$, lo cuál es una contradicción.

Recíprocamente, sea $x \in \bar{A}$, esto es, para todo $\epsilon>0$ se tiene $\mathbb{B}_{\epsilon}(x) \cap A \neq \emptyset$, en particular, para todo $n \in \mathbb{N}$ se tiene $\mathbb{B}_{\frac{1}{n}}(x) \cap A \neq \emptyset$. Lo que indica que, $d(x, A)<\frac{1}{n} \mathrm{y}$

$$
\lim _{n \rightarrow \infty} \frac{1}{n}=0
$$

entonces $d(x, A)=0$.

Definición 2.2. Sean $A$ y $B$ dos subconjuntos acotados y no vacíos de $\mathbb{R}^{n}$, la separación de Hausdorff de $B$ a $A$ es

$$
d_{H}^{*}(B, A)=\sup \{d(b, A): b \in B\} .
$$

Este concepto tiene una definición equivalente, $d_{H}^{*}(B, A)=$ ínf $\left\{\epsilon>0: B \subseteq A+\epsilon \overline{\mathbb{B}}_{1}(0)\right\}$, aparece en [2] su demostración, donde $\overline{\mathbb{B}}_{1}(0)$ es la bola cerrada de centro en 0 y radio 1 de $\mathbb{R}^{n}$.

Proposición 2.2. Sean $A, B, C \subseteq \mathbb{R}^{n}$ no vacíos y acotados, entonces

1. $d_{H}^{*}(B, A) \geq 0$

2. $d_{H}^{*}(B, A)=0$ si y solo si $B \subseteq \bar{A}$

3. $d_{H}^{*}(B, A) \leq d_{H}^{*}(B, C)+d_{H}^{*}(C, A)$

Prueba.

1. Como $d(b, A) \geq 0$ para todo $b \in B$, por propiedades del supremo (ver [1]),

$$
\sup \{d(b, A): b \in B\} \geq 0
$$

en consecuencia $d_{H}^{*}(B, A) \geq 0$.

2. Se supone que $d_{H}^{*}(B, A)=0$, esto es, $\sup \{d(b, A): b \in B\}=0, d(b, A)=0$ para todo $b \in B$; por la proposición $2.1 b \in \bar{A}$ y como es para todo elemento de $B$, se obtiene que $B \subseteq \bar{A}$.

Recíprocamente, si $B \subseteq \bar{A}$, por la proposición 2.1,

$$
d(b, A)=0 \text { para todo } b \in B
$$

en general, $\sup \{d(b, A): b \in B\}=0$, luego $d_{H}^{*}(B, A)=0$. 
3. Sean $d_{H}^{*}(B, C)=\alpha$ y $d_{H}^{*}(C, A)=\beta$, entonces $\alpha+\beta=d_{H}^{*}(B, C)+d_{H}^{*}(C, A)$, esto es,

$$
B \subseteq C+\alpha \overline{\mathbb{B}}_{1}(0) \text { y } C \subseteq A+\beta \overline{\mathbb{B}}_{1}(0)
$$

así,

$$
\begin{aligned}
B & \subseteq\left(A+\beta \overline{\mathbb{B}}_{1}(0)\right)+\alpha \overline{\mathbb{B}}_{1}(0) \\
& \subseteq A+(\beta+\alpha) \overline{\mathbb{B}}_{1}(0)
\end{aligned}
$$

con lo cual,

$$
\inf \left\{\epsilon>0: B \subseteq A+\epsilon \overline{\mathbb{B}}_{1}(0)\right\} \leq \alpha+\beta \text {. }
$$

En consecuencia, $d_{H}^{*}(B, A) \leq d_{H}^{*}(B, C)+d_{H}^{*}(C, A)$.

La separación de Hausdorff se constituye en un instrumento eficaz en la consecución de una métrica, claro está con algunas propiedades adicionales en el contexto.

Definición 2.3. Sean $A$ y $B$ dos subconjuntos no vacíos y acotados de $\mathbb{R}^{n}$, la distancia Hausdorff entre $A$ y $B$ es

$$
d_{H}(A, B)=\operatorname{máx}\left\{d_{H}^{*}(A, B), d_{H}^{*}(B, A)\right\} .
$$

Con esta definición, la distancia Hausdorff satisface la simetría, pero aún falta agregar condiciones adicionales al ambiente para obtener la estructura de espacio métrico; así, se restringe, aun más, la naturaleza de los subconjuntos de $\mathbb{R}^{n}$ en consideración. El resultado que sigue, se aplica a un universo específico con alguna incidencia en los demás.

Proposición 2.3. $\left(K^{n}, d_{H}\right)$, la colección de subconjuntos compactos de $\mathbb{R}^{n}$ con la distancia de Hausdorff, es un espacio métrico.

Prueba. Sean $A, B, C \subseteq \mathbb{K}^{n}$ no vacíos.

1. Se afirma que $d_{H}^{*}(A, B) \geq 0$ y $d_{H}^{*}(B, A) \geq 0$, entonces máx $\left\{d_{H}^{*}(A, B), d_{H}^{*}(B, A)\right\} \geq 0$, luego $d_{H}(A, B) \geq 0$

2. Sea $d_{H}(A, B)=0$, por propiedades del máximo $d_{H}^{*}(A, B)=0$ y $d_{H}^{*}(B, A)=0$, se tiene, $A \subseteq \bar{B}$ y $B \subseteq \bar{A}$, dado que $A$ y $B$ son cerrados de $\mathbb{R}^{n}$ entonces $A=\bar{A}$ y $B=\bar{B}$, es decir, $A=B$.

Recíprocamente, sea $A=B$, dado que $A, B$ son subconjuntos cerrados de $\mathbb{R}^{n}, A=\bar{A}$ y $B=\bar{B}$, de modo que $\bar{A}=\bar{B}$, esto es, $A \subseteq \bar{B}$ y $B \subseteq \bar{A}$, se tiene, $d_{H}^{*}(A, B)=0$ y $d_{H}^{*}(B, A)=0$, luego $d_{H}(A, B)=0$.

3. Se tiene que

$$
\begin{aligned}
d_{H}(A, B) & =\operatorname{máx}\left\{d_{H}^{*}(A, B), d_{H}^{*}(B, A)\right\} \\
& =\operatorname{máx}\left\{d_{H}^{*}(B, A), d_{H}^{*}(A, B)\right\} \\
& =d_{H}(B, A) .
\end{aligned}
$$


4. Como $d_{H}^{*}(B, A) \leq d_{H}^{*}(B, C)+d_{H}^{*}(C, A)$ y $d_{H}^{*}(A, B) \leq d_{H}^{*}(A, C)+d_{H}^{*}(C, B)$, entonces

$$
\begin{aligned}
d_{H}(A, B) & =\operatorname{máx}\left\{d_{H}^{*}(A, B), d_{H}^{*}(B, A)\right\} \\
& \leq \operatorname{máx}\left\{d_{H}^{*}(A, C)+d_{H}^{*}(C, B), d_{H}^{*}(B, C)+d_{H}^{*}(C, A)\right\} \\
& \leq \operatorname{máx}\left\{d_{H}^{*}(A, C), d_{H}^{*}(C, A)\right\}+\operatorname{máx}\left\{d_{H}^{*}(C, B), d_{H}^{*}(B, C)\right\} \\
& =d_{H}(A, C)+d_{H}(C, B)
\end{aligned}
$$

De modo que la distancia de Hausdorff mide cuan lejos están uno de otro dos subconjuntos compactos de $\mathbb{R}^{n}$. Además, $\left(K^{n}, d_{H}\right)$ cuenta con las propiedades de la completitud y $K_{C}^{n}$, la colección de subconjuntos compactos y convexos de $\mathbb{R}^{n}$, es un subconjunto cerrado en él; para ver esto, es necesario el resultado que sigue,

Proposición 2.4. Sean $A, B$ subconjuntos no vacios de $K^{n}$, si $d_{H}(A, B) \leq \epsilon \mathrm{y} b \in B$, existe $a \in A$ tal que $\|a-b\| \leq \epsilon$

Prueba. Sean $d_{H}(A, B) \leq \epsilon \mathrm{y} b \in B$, supongamos que para todo $a \in A$ se tiene $\|b-a\|>\epsilon$, en consecuencia ínf $\{\|a-b\|: a \in A\}>\epsilon$, es decir, $d(b, A)>\epsilon$. Por otro lado, $d_{H}(A, B) \leq \epsilon$, con lo cual $d_{H}^{*}(B, A) \leq \epsilon$, por tanto, $B \subseteq A+\epsilon \mathbb{B}_{1}(0)$. Así, para todo $b \in B$, se tiene que $d(b, A) \leq \epsilon$, lo que contradice el supuesto.

La prueba del siguiente teorema aparece en [2].

Teorema 2.1. $\left(K^{n}, d_{H}\right)$ es un espacio métrico completo ${ }^{1}$, además si $\left\{A_{n}\right\}_{n \in \mathbb{N}}$ es una sucesión de Cauchy en $K^{n}$, su límite es

$$
A=\left\{a \in \mathbb{R}^{n}: \text { existe }\left\{a_{n_{i}}\right\}_{i \in \mathbb{N}}, a_{n_{i}} \in A_{n_{i}} \text { y } \operatorname{lím}_{i \rightarrow \infty} a_{n_{i}}=a\right\} .
$$

Teorema 2.2. $K_{C}^{n}$, la colección de todos los conjuntos convexos compactos de $\mathbb{R}^{n}$, es un subconjunto cerrado del espacio métrico $\left(K^{n}, d_{H}\right)$.

Prueba. Sea $A \in K^{n}-K_{C}^{n}$, así $A$ es compacto no convexo, luego existen $x, y \in A$ y $\lambda \in[0,1]$ tales que $z=\lambda x+(1-\lambda) y \notin A$. Como $A$ es compacto, es cerrado, con lo cual existe $\epsilon>0$ tal que $\overline{\mathbb{B}}_{2 \epsilon}(z) \cap A=\emptyset$.

Sea $A^{\prime} \in K^{n}$ con $d_{H}\left(A, A^{\prime}\right)<\epsilon$, entonces, $A \subseteq A^{\prime}+\epsilon \overline{\mathbb{B}}_{1}(0)$ y como $x, y \in A$, existen $x^{\prime}, y^{\prime} \in A^{\prime}$ con $\left\|x-x^{\prime}\right\| \leq \epsilon \mathrm{y}\left\|y-y^{\prime}\right\| \leq \epsilon$. Luego:

$$
\begin{aligned}
\left\|\lambda x^{\prime}+(1-\lambda) y^{\prime}-z\right\| & =\left\|\lambda x^{\prime}+(1-\lambda) y^{\prime}-\lambda x+(1-\lambda) y\right\| \\
& \leq \lambda\left\|x-x^{\prime}\right\|+(1-\lambda)\left\|y-y^{\prime}\right\| \\
& \leq \lambda \epsilon+(1-\lambda) \epsilon \\
& =\epsilon
\end{aligned}
$$

Ahora sea $z^{\prime}=\lambda x^{\prime}+(1-\lambda) y^{\prime}$ y se supone que $z^{\prime} \in A^{\prime}$, como $A^{\prime} \subseteq A+\epsilon \overline{\mathbb{B}}_{1}(0)$, existe $w \in A$ tal que $\left\|z^{\prime}-w\right\| \leq \epsilon$. Entonces $\|z-w\| \leq\left\|z-z^{\prime}\right\|+\left\|z^{\prime}-w\right\| \leq 2 \epsilon$. Lo que contradice que

\footnotetext{
${ }^{1}$ Sea $(X, d)$ un espacio métrico, si toda sucesión de Cauchy en $X$ converge, se dice que $(X, d)$ es un espacio métrico completo.
} 
$\overline{\mathbb{B}}_{2 \epsilon}(z) \cap A=\emptyset$. Luego $A^{\prime}$ es no convexo y por tanto $A$ es un conjunto abierto de $\left(K^{n}, d_{H}\right)$.

Con esto, $\left(K_{C}^{n}, d_{H}\right)$ es también un espacio métrico completo [1]. El siguiente resultado aparece en [8], está dado para elementos de $K_{C}^{n}$, sin embargo en [6], se observa que para elementos de $K^{n}$ funciona igualmente.

Proposición 2.5. Sea $\left\{K_{m}\right\}_{m \in \mathbb{N}}$ una sucesión en $K_{C}^{n}$ que converge a $K$, además, sea

$$
\lim _{m \rightarrow \infty} d_{H}\left(K_{m}, K\right)=0
$$

entonces,

$$
K=\bigcap_{i \geq 1} \overline{\bigcup_{m \geq i} K_{m}}
$$

Prueba. Sea $\epsilon>0, n \in \mathbb{N}$ y $x \in K$, entonces existe $m \geq n$ tal que $d_{H}\left(K_{m}, K\right) \leq \epsilon$, por la proposición 2.4, existe un punto $x_{m} \in K_{m}$ tal que $\left\|x-x_{m}\right\| \leq 2 \epsilon$. Por consiguiente, $x \in$ $\overline{\bigcup_{m \geq n} K_{m}}$ para cada $n \in \mathbb{N}$.

Sea $x \in \bigcap_{i \geq 1} \overline{\bigcup_{m \geq i} K_{m}}$, entonces para cada $p \in \mathbb{N}$ existe $m \geq p$ tal que $d\left(x, K_{m}\right) \leq \epsilon$. Así, si $n \geq p$, se tiene que:

$$
d\left(x, K_{n}\right) \leq d\left(x, K_{m}\right)+d_{H}\left(K, K_{m}\right) \leq 2 \epsilon
$$

entonces $x \in \bigcap_{i \geq 1} \overline{\bigcup_{m \geq i} K_{m}}$ para $n$, esto prueba que $K \subseteq \bigcap_{i \geq 1} \overline{\bigcup_{m \geq i} K_{m}}$.

Sea $x \in \bigcap_{i \geq 1} \overline{\bigcup_{m \geq i} K_{m}}$, de que la sucesión $\left\{K_{m}\right\}$ converge a $K$, se tiene que

$$
d_{H}^{*}\left(K_{m}, K \cup\{x\}\right)=0 \text { cuando } m \rightarrow \infty,
$$

además sea $p$ tal que para $m, n \geq p$ implique $d_{H}\left(K_{n}, K_{m}\right) \leq \epsilon$. Del hecho que $x \in \bigcap_{i \geq 1} \overline{\bigcup_{m \geq i} K_{m}}$ se sigue que existe $m \geq p$ tal que $d\left(x, K_{m}\right) \leq \epsilon$, luego si $n \geq p$

$$
d\left(x, A_{n}\right) \leq d\left(x, K_{m}\right)+d_{H}\left(K_{m}, K_{n}\right) \leq 2 \epsilon,
$$

con lo cual $d_{H}\left(K_{n}, K \cup\{x\}\right)=0$ y luego $\bigcap_{i \geq 1} \overline{\bigcup_{m \geq i} K_{m}} \subseteq K$.

\section{El espacio $E^{n}$}

Definición 3.1. Un subconjunto difuso $u$ de $X$, está determinado por una función $u: X \rightarrow[0,1]$, que indica el grado de pertenencia o membresía de un elemento $x$ en el conjunto $u$.

Es de aclarar, que en la teoría difusa se utilizan diferentes tipos de notaciones, en este caso, se siguen las ideas de [12], pero dado a las modificaciones que con el tiempo se han venido utilizando, se sigue la notación de [5].

Obsérvese que $u$ generaliza la noción de función característica de un conjunto. Además, de acuerdo con [5], si $u(x)=0, x$ no pertenece al conjunto; si $u(x)=1, x$ pertenece al conjunto y si $0<u(x)<1$, se tiene que $x$ pertenece de manera parcial, su grado de membresía es justamente $u(x)$ 
Un subconjunto $A$ de $B$, se caracteriza, por tanto, por la función de pertenencia $A: B \rightarrow[0,1]$, es preciso fijar el conjunto $B$ para definir la función $A$, que a su vez define $A$. Por eso se habla de subconjunto difuso y no de conjunto difuso, (otros detalles en [12]); Ahora se presta atención a una colección particular de subconjuntos difusos de $\mathbb{R}^{n}$.

Sea $E^{n}$ la colección de todos los subconjuntos difusos de $\mathbb{R}^{n}$ que satisfacen:

1. El soporte y los $\alpha$-cortes $^{3}$ de $u$ son conjuntos compactos de $\mathbb{R}^{n}$, para todo $\alpha \in[0,1]$,

2. $u$ es convexo difuso, esto es, para $x, y \in \mathbb{R}^{n}$

$$
u(\lambda x+(1-\lambda) y) \geq \operatorname{mín}\{u(x), u(y)\}
$$

para todo $\lambda \in[0,1]$.

Lema 3.1. Si $u \in E^{n}$, entonces se satisface

$$
\begin{gathered}
{[u]^{\alpha} \in K_{C}^{n} \text { para todo } \alpha \in[0,1],} \\
{[u]^{\alpha_{2}} \subseteq[u]^{\alpha_{1}} \text { para } 0 \leq \alpha_{1} \leq \alpha_{2} \leq 1}
\end{gathered}
$$

y si $\left\{\alpha_{k}\right\}_{k \in \mathbb{N}}$ es una sucesión creciente que converge a $\alpha$, entonces

$$
[u]^{\alpha}=\bigcap_{k \geq 1}[u]^{\alpha_{k}}
$$

Recíprocamente, si $\left\{A^{\alpha}: 0 \leq \alpha \leq 1\right\}$ es la colección de subconjuntos de $\mathbb{R}^{n}$ que satisface (1), (2) y (3), entonces existe un $u \in E^{n}$ tal que

$$
[u]^{\alpha}=A^{\alpha} \text { para } 0<\alpha \leq 1
$$

$\mathrm{y}$

$$
[u]^{0}=\overline{\bigcup_{0<\alpha \leq 1} A^{\alpha}} \subset A^{0} .
$$

Prueba. Sea $u \in E^{n}$, por la definición de $E^{n},[u]^{\alpha}$ es compacto para $\alpha \in[0,1]$, resta entonces ver que $[u]^{\alpha}$ es convexo. Para $\alpha \in(0,1]$, sean $x, y \in[u]^{\alpha}$, esto es, $u(x) \geq \alpha$ y $u(y) \geq \alpha$, entonces al ser $u$ convexo difuso se tiene

$$
u(\lambda x+(1-\lambda) y) \geq \operatorname{mín}\{u(x), u(y)\} \geq \alpha,
$$

luego $(\lambda x+(1-\lambda) y) \in[u]^{\alpha}$. Con lo cual se satisface (1).

Sean $0 \leq \alpha_{1} \leq \alpha_{2} \leq 1$, se sabe que

$$
\begin{aligned}
{[u]^{\alpha_{2}} } & =\left\{x \in \mathbb{R}^{n}: u(x) \geq \alpha_{2}\right\} \\
& \subseteq\left\{x \in \mathbb{R}^{n}: u(x) \geq \alpha_{1}\right\} \\
& =[u]^{\alpha_{1}}
\end{aligned}
$$

con lo que se satisface (2).

Ahora sea $\left\{\alpha_{k}\right\}_{k \in \mathbb{N}}$ una sucesión creciente que converge a $\alpha$, luego por (2), se tiene que

${ }^{2}$ Para un conjunto difuso $u$ de $\mathbb{R}^{n}$, el soporte de $u$ es $[u]^{0}=\overline{\bigcup_{\alpha \in(0,1]}[u]^{\alpha}}[5]$

${ }^{3}$ Para un conjunto difuso $u$ de $\mathbb{R}^{n}$, el $\alpha$-corte es $[u]^{\alpha}=\{x \in X: u(x) \geq \alpha\}$ para $\alpha \in(0,1]$ [5] 


$$
[u]^{\alpha_{1}} \subseteq[u]^{\alpha_{2}} \subseteq \ldots \subseteq[u]^{\alpha_{k}} \subseteq \ldots \subseteq[u]^{\alpha}
$$

por otro lado, $[u]^{\alpha_{k}} \in K_{C}^{n}$, ahora aplicando la proposición 2,9, se tiene que esta sucesión converge a $A=\bigcap_{k \geq 1}[u]^{\alpha_{k}}$, con lo cual $[u]^{\alpha}=\bigcap_{k \geq 1}[u]^{\alpha_{k}}$, y (3) se sigue.

Recíprocamente, sea $\left\{A^{\alpha}: 0 \leq \alpha \leq 1\right\}$ la colección de subconjuntos de $\mathbb{R}^{n}$ que satisface (1), (2) y (3); dado $x \in A^{0}$, se define $I_{x}=\left\{\alpha \in I: x \in A^{\alpha}\right\}$, y sea $\alpha_{0}=\sup I_{x}$, de donde se obtiene que $I_{x}=\left[0, \alpha_{0}\right]$. En efecto, si $\alpha_{0}=0$, de inmediato $I_{x}=\left[0, \alpha_{0}\right]$, entonces se supone que $\alpha_{0}>0$ y sea $\beta \in\left(0, \alpha_{0}\right)$; luego existe $\beta_{1} \in\left[\beta, \alpha_{0}\right)$ tal que $\beta_{1} \in I_{x}$, así, dado que $x \in A^{\beta_{1}}$ implica por (2), $x \in A^{\beta}$ y $\beta \in I_{x}$, por definición $0 \in I_{x}$ y se obtiene que $\left[0, \alpha_{0}\right) \subseteq I_{x}$; ahora sea $\left\{\alpha_{i}\right\}_{i \in \mathbb{N}}$ una sucesión monotona en $I_{x}$ que converge a $\alpha_{0}$, entonces $x \in A^{\alpha_{i}}$ para cada $i=1,2, \ldots$ y por (3), $x \in A^{\alpha_{0}}$, así $\alpha_{0} \in I_{x}$ y $\left[0, \alpha_{0}\right] \subseteq I_{x}$. Igualmente, dado $\beta \in I_{x}$ implica que $\beta \leq \alpha_{0}$, luego $I_{x} \subseteq\left[0, \alpha_{0}\right]$.

Se define $u: \mathbb{R}^{n} \rightarrow[0,1]$ como $u(x)=\sup I_{x}$ para todo $x \in \mathbb{R}^{n}$, por consiguiente, sea $\alpha \in(0,1]$, si $x \in[u]^{\alpha}$ entonces $u(x) \geq \alpha>0$, así $x \in A^{0}$ y $u(x)=\sup I_{x}=\alpha_{0} \geq 0$; por consiguiente, $x \in A^{\alpha}$ y por $2, x \in A^{\alpha}$, esto es, $[u]^{\alpha} \subseteq A^{\alpha}$. Si $x \in A^{\alpha}$, entonces $u(x)=\sup I_{x}=\alpha_{0} \geq \alpha \mathrm{y}$ $x \in[u]^{\alpha}$. Por tanto $[u]^{\alpha}=A^{\alpha}$.

Construido lo anterior, se verifica que $u \in E^{n}$, en efecto, $u$ es un conjunto difuso de $\mathbb{R}^{n}$ por su definición, ahora $[u]^{\alpha}=A^{\alpha} \in K_{C}^{n}$ por (1), entonces $[u]^{\alpha}$ es compacto para todo $\alpha \in[0,1]$. Finalmente, sean $x, y \in[u]^{\alpha}$ con mín $[u(x), u(y)]=\gamma \geq \alpha$, entonces $x, y \in[u]^{\gamma}$, que es convexo y así $\lambda x+(1-\lambda) y \in[u]^{\gamma}$ para cualquier $\lambda \in[0,1]$. Por consiguiente

$$
u(\lambda x+(1-\lambda) y) \geq \gamma=\min [u(x), u(y)]
$$

lo que prueba que $u$ es convexo difuso.

Al espacio $E^{n}$ se le puede dotar una estructura para sus $\alpha$-cortes, es expuesto con el siguiente concepto, cabe resaltar que los dos anteriores resultados son tomados de [8].:

Lema 3.2. Sean $u, v \in E^{n}$ entonces la adición ${ }^{4} u \widetilde{+} v$ y multiplicación por un escalar ${ }^{5} \widetilde{c u}$ difusa pertenecen a $E^{n}$, donde los $\alpha$-cortes son definidos como:

$$
[u \tilde{+} v]^{\alpha}=[u]^{\alpha}+[v]^{\alpha}
$$

$\mathrm{y}$

$$
[\widetilde{c u}]^{\alpha}=c[u]^{\alpha}
$$

para $c \in \mathbb{R}-\{0\}$.

Prueba. Dado que $u, v \in E^{n}$, se satisfacen (1), (2) y (3), entonces $[u \tilde{+} v]^{\alpha} \in K_{C}^{n}$; sean $0 \leq \alpha_{1} \leq$ $\alpha_{2} \leq 1$, entonces

\footnotetext{
${ }^{4}$ Dado que la suma de dos subconjuntos difusos no es clausurativa, por medio del principio de extensión de Zadeh se define la suma difusa $u \tilde{+} v: \mathbb{R}^{n} \rightarrow[0,1] \operatorname{como}(u \tilde{+} v)(x)=\sup \left\{\operatorname{mín}\left\{u\left(x_{2}\right), v\left(x_{1}\right)\right\}: x=x_{1}+x_{2}\right\}$ [12].

${ }^{5}$ La multiplicación por escalar $c \in \mathbb{R}^{n}-\{0\}$ difusa $\widetilde{c v}: \mathbb{R}^{n} \rightarrow[0,1]$ es definida según el principio de extensión de Zadeh como $(\widetilde{c v})(x)=u\left(\frac{x}{c}\right)[12]$.
} 


$$
[u \tilde{+} v]^{\alpha_{2}}=[u]^{\alpha_{2}}+[v]^{\alpha_{2}} \subseteq[u]^{\alpha_{1}}+[v]^{\alpha_{1}}=[u \tilde{+} v]^{\alpha_{1}}
$$

sea $\left\{\alpha_{i}\right\}_{i \in \mathbb{N}}$ una sucesión creciente que converge a $\alpha>0$ entonces

$$
\begin{aligned}
d_{H}\left([u \tilde{+} v]^{\alpha_{i}},[u \tilde{+} v]^{\alpha}\right) & =d_{H}\left([u]^{\alpha_{i}}+[v]^{\alpha_{i}},[u]^{\alpha}+[v]^{\alpha}\right) \\
& \leq d_{H}\left([u]^{\alpha_{i}},[u]^{\alpha}\right)+d_{H}\left([v]^{\alpha_{i}},[v]^{\alpha}\right)
\end{aligned}
$$

de donde $\lim _{i \rightarrow \infty} d_{H}\left([u \widetilde{+} v]^{\alpha_{i}},[u \widetilde{+} v]^{\alpha}\right)=0$, luego, por la proposición 2.5, $[u \widetilde{+} v]^{\alpha}=\bigcap_{k \geq 1}[u]^{\alpha_{k}}$. De modo que $\left\{[u \widetilde{+} v]^{\alpha}: \alpha \in I\right\}$ satisface (1), (2) y (3), por el teorema 3.1, $u \widetilde{+} v \in E^{n}$.

Similarmente, $[\widetilde{c u}]^{\alpha} \in K_{C}^{n}$; sean $0 \leq \alpha_{1} \leq \alpha_{2} \leq 1$, entonces

$$
[\widetilde{c u}]^{\alpha_{2}}=c[u]^{\alpha_{2}} \subseteq c[u]^{\alpha_{1}}=[\widetilde{c u}]^{\alpha_{1}}
$$

sea $\left\{\alpha_{i}\right\}_{i \in \mathbb{N}}$ una sucesión creciente que converge a $\alpha>0$ entonces

$$
\begin{aligned}
d_{H}\left([\widetilde{c u}]^{\alpha_{i}},[\widetilde{c u}]^{\alpha}\right) & =d_{H}\left(c[u]^{\alpha_{i}}, c[u]^{\alpha}\right) \\
& \leq|c| d_{H}\left([u]^{\alpha_{i}},[u]^{\alpha}\right)
\end{aligned}
$$

de donde $\operatorname{lím}_{i \rightarrow \infty} d_{H}\left([\widetilde{c u}]^{\alpha_{i}},[\widetilde{c u}]^{\alpha}\right)=0$, luego por la proposición $2.5,[\widetilde{c u}]^{\alpha}=\bigcap_{k \geq 1}[u]^{\alpha_{k}}$. De modo que $\left\{[\widetilde{c u}]^{\alpha}: \alpha \in I\right\}$ satisface (1), (2) y (3), por el teorema 3.1, $\widetilde{c u} \in E^{n}$.

\section{El espacio métrico $\left(E^{n}, d\right)$}

Se procede al intersectar las dos teorías expuestas, se tiene el siguiente resultado, que ha sido difundido por variados autores, entre ellos V. Lakshmikantham em [8].

Lema 4.1. El par $\left(E^{n}, d\right)$ con la métrica del supremo $d$ en $E^{n}$ definida como

$$
d(u, v)=\sup \left\{d_{H}\left([u]^{\alpha},[v]^{\alpha}\right): \alpha \in[0,1]\right\}
$$

donde $u, v \in E^{n}$, es un espacio métrico.

Prueba. Sean $u, v, w \in E^{n}$, entonces

1. Para cada $\alpha \in[0,1]$, dado que $d_{H}\left([u]^{\alpha},[v]^{\alpha}\right) \geq 0$, luego por propiedades del supremo se obtiene que:

$$
d(u, v)=\sup \left\{d_{H}\left([u]^{\alpha},[v]^{\alpha}\right): \alpha \in[0,1]\right\} \geq 0
$$

2. Sea $d(u, v)=0$, entonces $\sup \left\{d_{H}\left([u]^{\alpha},[v]^{\alpha}\right): \alpha \in[0,1]\right\}=0$, por propiedades del supremo,

$$
d_{H}\left([u]^{\alpha},[v]^{\alpha}\right)=0
$$


para todo $\alpha \in[0,1]$, luego $[u]^{\alpha}=[v]^{\alpha}$, entonces $u=v$.

Recíprocamente, si $u=v$ entonces $[u]^{\alpha}=[v]^{\alpha}$, luego

$$
d_{H}\left([u]^{\alpha},[v]^{\alpha}\right)=0
$$

así por propiedades del supremo, $\sup \left\{d_{H}\left([u]^{\alpha},[v]^{\alpha}\right): \alpha \in[0,1]\right\}=0, \operatorname{luego} d(u, v)=0$.

3. La simetría se satisface, en efecto,

$$
\begin{aligned}
d(u, v) & =\sup \left\{d_{H}\left([u]^{\alpha},[v]^{\alpha}\right): \alpha \in[0,1]\right\} \\
& =\sup \left\{d_{H}\left([v]^{\alpha},[u]^{\alpha}\right): \alpha \in[0,1]\right\} \\
& =d(v, u)
\end{aligned}
$$

4. Ahora se verifica la desigualdad triangular,

$$
\begin{aligned}
d(u, v) & =\sup \left\{d_{H}\left([u]^{\alpha},[v]^{\alpha}\right): \alpha \in[0,1]\right\} \\
& \leq \sup \left\{d_{H}\left([u]^{\alpha},[w]^{\alpha}\right)+d_{H}\left([w]^{\alpha},[v]^{\alpha}\right): \alpha \in[0,1]\right\} \\
& \leq \sup \left\{d_{H}\left([u]^{\alpha},[w]^{\alpha}\right): \alpha \in[0,1]\right\}+\sup \left\{d_{H}\left([w]^{\alpha},[v]^{\alpha}\right): \alpha \in[0,1]\right\} \\
& =d(u, w)+d(w, v) .
\end{aligned}
$$

Finalmente, se prueba:

Teorema 4.1. $\left(E^{n}, d\right)$ es un espacio métrico completo

Prueba. Sea $\left\{u_{k}\right\}$ una sucesión de Cauchy en $\left(E^{n}, d\right)$, entonces $\left\{\left[u_{k}\right]^{\alpha}\right\}$ para cada $\alpha \in[0,1]$, es una sucesión de Cauchy in $\left(K_{C}^{n}, d_{H}\right)$, que es un espacio métrico completo, así, existe un $C_{\alpha} \in K_{C}^{n}$ para cada $\alpha \in[0,1]$ tal que

$$
\lim _{k \rightarrow \infty} d_{H}\left(\left[u_{k}\right]^{\alpha}, C_{\alpha}\right)=0
$$

Se considera la colección $\left\{C_{\alpha}: \alpha \in[0,1]\right\}$, y cada $C_{\alpha} \in K_{C}^{n}$ lo que satisface (1); sea $0 \leq \beta<$ $\alpha \leq 1$, entonces

$$
\begin{aligned}
d_{H}^{*}\left(C_{\alpha}, C_{\beta}\right) & \leq d_{H}^{*}\left(C_{\alpha},\left[u_{k}\right]^{\alpha}\right)+d_{H}^{*}\left(\left[u_{k}\right]^{\alpha},\left[u_{k}\right]^{\beta}\right)+d_{H}^{*}\left(\left[u_{k}\right]^{\beta}, C_{\beta}\right) \\
& =d_{H}^{*}\left(C_{\alpha},\left[u_{k}\right]^{\alpha}\right)+d_{H}^{*}\left(\left[u_{k}\right]^{\beta}, C_{\beta}\right) \\
& \leq d_{H}\left(C_{\alpha},\left[u_{k}\right]^{\alpha}\right)+d_{H}\left(C_{\beta},\left[u_{k}\right]^{\beta}\right)
\end{aligned}
$$

con lo cual

$$
d_{H}^{*}\left(C_{\alpha}, C_{\beta}\right)=0
$$

y esto indica, segun la proposición 2.2, que $C_{\alpha} \subseteq C_{\beta}$, luego se satisface (2); sea $\left\{\alpha_{i}\right\}$ una sucesión decreciente en $[0,1]$ que converge a $\alpha$, con lo cual $C_{\alpha} \subseteq C_{\alpha_{i}}$ para $i=1,2,3, \ldots$, luego, 


$$
C_{\alpha} \subseteq \bigcap_{i=1} C_{\alpha_{i}}
$$

ahora sea $x \in \bigcap_{i=1} C_{\alpha_{i}}$, así, $x \in C_{\alpha_{i}}$ para todo $i=1,2,3, \ldots$, entonces

$$
\begin{aligned}
d_{H}^{*}\left(\{x\}, C_{\alpha}\right) & \leq d_{H}^{*}\left(\{x\}, C_{\alpha_{i}}\right)+d_{H}^{*}\left(C_{\alpha_{i}}, C_{\alpha}\right) \\
& =d_{H}^{*}\left(C_{\alpha_{i}}, C_{\alpha}\right) \\
& \leq d_{H}^{*}\left(C_{\alpha_{i}},\left[u_{k}\right]^{\alpha_{i}}\right)+d_{H}^{*}\left(\left[u_{k}\right]^{\alpha_{i}},\left[u_{k}\right]^{\alpha}\right)+d_{H}^{*}\left(\left[u_{k}\right]^{\alpha}, C_{\alpha}\right),
\end{aligned}
$$

de modo que $d_{H}^{*}\left(\{x\}, C_{\alpha}\right)=0$, por la proposición 2.2, $x \in C_{\alpha}$ y por consiguiente

$$
\bigcap_{i=1} C_{\alpha_{i}} \subseteq C_{\alpha}
$$

Por tanto, se satisface la condición (3) de que

$$
C_{\alpha}=\bigcap_{i=1} C_{\alpha_{i}}
$$

Entonces al satisfacer (1),(2) y (3), se aplica el teorema 3.1, con lo cual, existe un $u \in E^{n}$ tal que $[u]^{\alpha}=C_{\alpha}$ para todo $\alpha \in[0,1]$. Además,

$$
\begin{aligned}
d_{H}\left(\left[u_{k}\right]^{\alpha},[u]^{\alpha}\right) & \leq d_{H}\left(\left[u_{k}\right]^{\alpha},\left[u_{j}\right]^{\alpha}\right)+d_{H}\left(\left[u_{j}\right]^{\alpha},[u]^{\alpha}\right) \\
& \leq\left\|u_{k}-u_{j}\right\|+d_{H}\left(\left[u_{j}\right]^{\alpha},[u]^{\alpha}\right) \\
& <\epsilon+d_{H}\left(\left[u_{j}\right]^{\alpha},[u]^{\alpha}\right)
\end{aligned}
$$

para todo $j, k \geq N(\epsilon)$, debido a que $\left\{u_{k}\right\}$ es una sucesión de Cauchy en $\left(E^{n}, d\right)$. Tomando el límite cuando $j \rightarrow \infty$, se obtiene

$$
d_{H}\left(\left[u_{k}\right]^{\alpha},[u]^{\alpha}\right) \leq \epsilon
$$

para todo $k \geq N(\epsilon)$ y $\alpha \in I$, por tanto $d\left(u, u_{k}\right) \leq \epsilon$ para todo $k \geq N(\epsilon)$ y $\left(E^{n}, d\right)$ es un espacio métrico completo.

Los conceptos arriba expuestos, se extienden y profundizan en [8], [9] y [10] en donde el horizonte se amplía y abre lejanas perspectivas.

\section{Comparación con otros espacios métricos}

De las secciones anterior resultó un nuevo espacio métrico, la idea ahora es realizar una comparación con otros espacios métricos [11] relacionados con subconjuntos difusos.

Sean $A, B$ subconjuntos difusos de un universo $X$ cualquiera, la distancia de Hamming se define como

$$
d(A, B)=\int_{x}|A(x)-B(x)|, d x,
$$


la distancia euclídea como

$$
d(A, B)=\sqrt{\int_{x}|A(x)-B(x)|^{2} d x},
$$

y la distancia de Tchebyschev como

$$
d(A, B)=\sup _{x \in X}|A(x)-B(x)| .
$$

Se resaltar que dichas distancias se encuentran entre dos subconjuntos difusos con el mismo universo $X$ y se puede afirmar que cuanto mayor sea la similitud de los subconjuntos difusos, la distancia es menor.

En el espacio $\left(E^{n}, d\right)$ solo intervienen subconjuntos difusos de $\mathbb{R}^{n}$ con restricciones particulares ya expuestas, para estas tres distancias, no hay restricción alguna, así que se obtiene una bifurcación de los subconjuntos difusos, y por consiguiente no estan muy relacionadas. Además la información obtenida de las tres distancias es muy débil, mostrando una cualidad muy general, que es grado de similaridad de dos subconjuntos dados. Mientras que en el espacio $\left(E^{n}, d\right)$, se comparam los $\alpha$-cortes con la métrica de Hausdorff, es decir, se le está dando analisis a cada $\alpha \in[0,1]$.

Se presenta una situación en donde se calculan las cuatro distancias. Sean $u, v \in E^{1}$, como las figuras 1 y 2, y matemáticamente definidos de la siguiente forma:

$$
u(x)=\left\{\begin{array}{ccc}
0 & \text { si } & -\infty<x<0 \\
1 & \text { si } & x=0 \\
\frac{1}{2} & \text { si } & 0<x \leq 1 \\
0 & \text { si } & 1<x<\infty
\end{array}\right.
$$

$\mathrm{y}$

$$
v(x)=\left\{\begin{array}{llc}
0 & \text { si } & -\infty<x<0 \\
\frac{-x}{2}+1 & \text { si } & 0 \leq x \leq 1 \\
0 & \text { si } & 1<x<\infty
\end{array}\right.
$$

De esta forma podemos decir que los $\alpha$-cortes son

$$
\begin{gathered}
{[u]^{\alpha}=[v]^{\alpha}=[0,1] \text { para } 0 \leq \alpha \leq \frac{1}{2},} \\
{[u]^{\alpha}=0 \text { para } \frac{1}{2}<\alpha \leq 1}
\end{gathered}
$$

$\mathrm{y}$

$$
[u]^{\alpha}=[v]^{\alpha}=[0,2(1-\alpha)] \text { para } \frac{1}{2}<\alpha \leq 1
$$




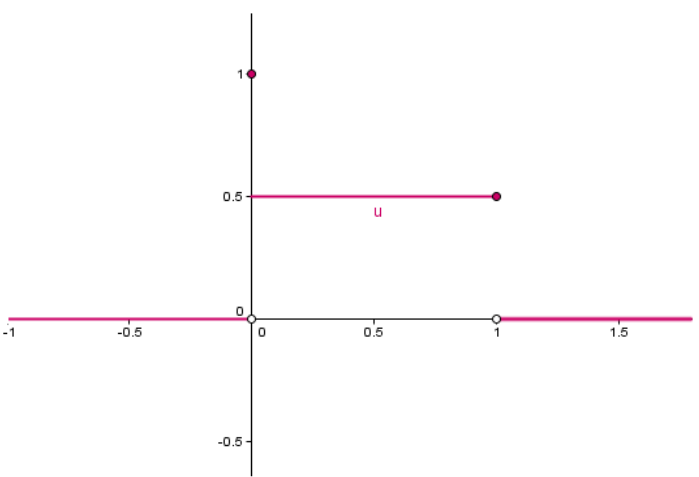

Figura 1. Representación gráfica del subconjunto difusos $u(x)$

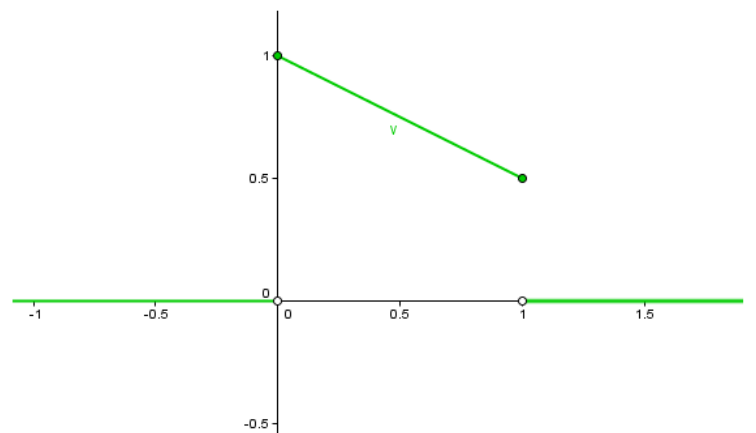

Figura 2. Representación gráfica del subconjuntos difusos $v(x)$

Luego se tiene que $d_{H}\left([u]^{\alpha},[v]^{\alpha}\right)=0$ para $\alpha \in\left[0, \frac{1}{2}\right]$ y $d_{H}\left([u]^{\alpha},[v]^{\alpha}\right)=2(1-\alpha)$ para $\alpha \in\left(\frac{1}{2}, 1\right]$. Por tanto $d(u, v)=1$.

Para las otras distancias se tienen los siguientes cálculos, para la distancia de Hamming

$$
d(A, B)=\int_{\mathbb{R}}|u(x)-v(x)| d x=\int_{0}^{1}\left|1-\left(\frac{-x}{2}+1\right)\right| d x=\frac{1}{4},
$$

para la distancia euclídea

$$
d(A, B)=\sqrt{\int_{\mathbb{R}}|u(x)-v(x)|^{2} d x}=\sqrt{\int_{0}^{1}\left|1-\left(\frac{-x}{2}+1\right)\right|^{2} d x}=\frac{\sqrt{3}}{6}
$$

y para la distancia de Tchebyschev

$$
d(A, B)=\sup _{x \in \mathbb{R}}|u(x)-v(x)|=\sup _{x \in[0,1]}\left|1-\left(\frac{-x}{2}+1\right)\right|=\frac{1}{2} .
$$

Por los gráficos se observa que los subconjuntos difusos son similares y esto se ve representado en las tres distancias, ya que se acercan a 0 . Mientras que con la métrica del espacio $E^{1}$, no tienen relación, al realizar los cálculos, es necesario el análisis de los $\alpha$-cortes, osea que, en casos de aplicaciones, se tendrá que analizar parte por parte del subconjunto difuso, proporcionando más información de su significado.

\section{Conclusiones}

La construcción de la métrica de Hausdorff en $\mathbb{R}^{n}$ es una edificación desde la definición de distancia entre un punto y un conjunto acotado no vacío, con ella se produce un nuevo espacio métrico completo $\left(K^{n}, d_{H}\right)$ con los subconjuntos compactos de $\mathbb{R}^{n}$, que además se obtiene $K_{C}^{n}$, el conjunto 
de compactos y convexos de $\mathbb{R}^{n}$, es un conjunto cerrado para este espacio métrico.

El espacio $E^{n}$ tiene dos operaciones cerradas de adición y multiplicación por un escalar entre sus elementos, dado por el principio de extensión de Zadeh y las propiedades del espacio.

Al relacionar el espacio $E^{n}$ y la métrica de Hausdorff, se enriquece la noción de la métrica de Hausdorff, obteniendo un espacio completo. Esta propiedad permite la utilidad en ambientes diferentes, lo que conlleva a otros rumbos de investigación.

Comparando con otras distancias entre subconjuntos difusos, se puede afirmar que en $\left(E^{n}, d\right)$ se necesita analizar cada elemento del espacio para poder obtener su distancia con otro, luego es necesario un mayor detalle y con eso conocer mejor su naturaleza.

\section{Referencias}

[1] T. M. Apostol. Mathematical Analysis. Addison-Wesley Publishing Company. Massachusetts. 1981.

[2] M. Barnsley. Fractals Everywhere. Academic Press. San Diego. 1988.

[3] P. Diamond y P. Kloeden. Metric Spaces of Fuzzy Sets. World Scientific. Singapore. 1994.

[4] D. Dubois y H. Prade. Fuzzy Sets and Systems: Theory and Applications. Academic Press. 1980.

[5] W. González. Una aproximación a los conjuntos alcanzables de una inclusión diferencial difusa. Revista Integración, vol. 30, núm. 1, 2012, pp. 57-74.

[6] C. Castaing y M. Valadier. Convex Analysis and Measurable Multifunctions. Springer-Verlag. 1932.

[7] E. Kreyszig Introductory Functional Analysis with Applications. John Wiley \& Sons. Canada 1978.

[8] V. Lakshmikanthan y R.N. Mohapatra. Theory of Fuzzy Differential Equantions and Inclusions. Taylor y Francis. 2003.

[9] J. Rodríguez-Lópeza y S. Romaguera. On completion of fuzzy metric spaces. Fuzzy Sets and Systems. Vol.130, 2002, pp.399-404-283.

[10] J. Rodríguez-Lópeza y S. Romaguera. The Hausdorff fuzzy metric on compact sets. Fuzzy Sets and Systems. Vol.147, 2004, pp.273-283.

[11] S. S. Silva. Modelo Basado en Lógica difusa para la comparación de objetos con atributos imprecisos. Universidad Centro Occidental Lisandro Alvarado. 2013.

[12] L. A. Zadeh. Fuzzy Sets. Inf. Control 8. 1965.

\section{Carlos Orlando Ochoa Castillo}

Normalista Superior; Licenciado en Educación con Especialidad en Matemáticas y Física; especialista en Matemática Aplicada; Magister Scientiae - Matemáticas; ha sido coordinador de los programas de Matemáticas y Licenciatura en Matemáticas de la Universidad Distrital Francisco José de Caldas.

\section{Laura Victoria Forero Vega}

Matemática de la Universidad Distrital Francisco José de Caldas; magíster (c) en matemática aplicada en la Universidad de São Paulo, Brasil. 